\title{
Interview with Abel Laureate Karen Uhlenbeck
}

\section{Bjørn Ian Dundas and Christian Skau}

Karen Uhlenbeck is the recipient of the 2019 Abel Prize of the Norwegian Academy of Science and Letters. ${ }^{1}$ The following interview originally appeared in the September 2019 issue of the Newsletter of the European Mathematical Society ${ }^{2}$ and is reprinted here with permission of the EMS.

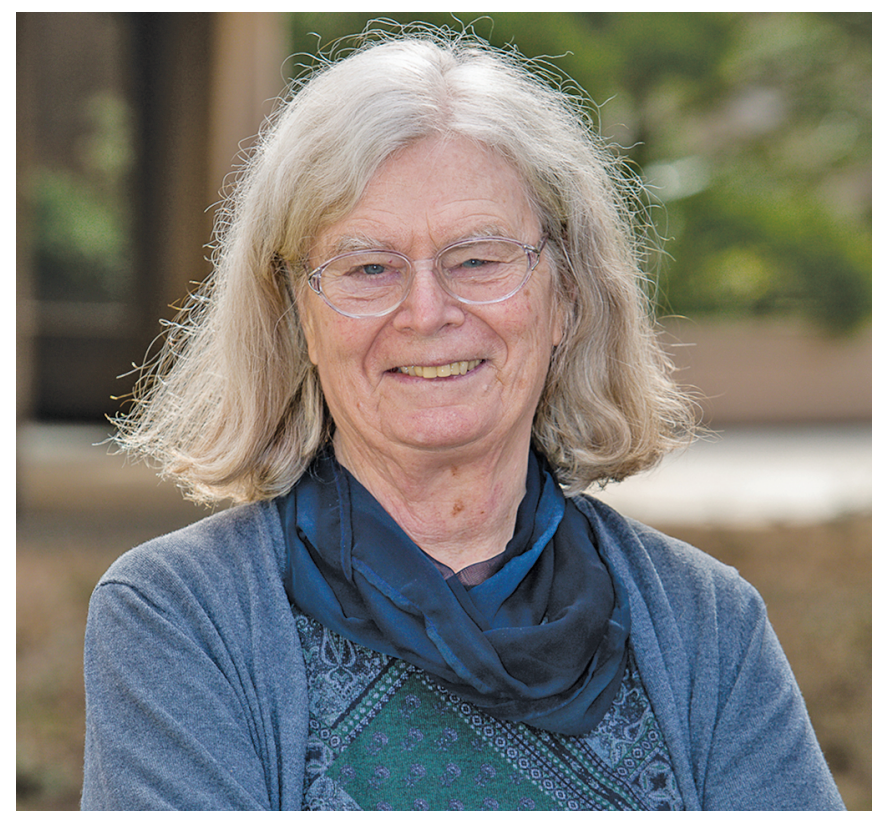

Figure 1. Karen Uhlenbeck, the Abel Prize Laureate 2019.

Bjørn Ian Dundas is a professor of mathematics at the University of Bergen, Norway. His email address is Bjorn.Dundas@uib.no.

Christian Skau is a professor emeritus of mathematics at the Norwegian University of Science and Technology, Trondheim, Norway. His email address is csk@math.ntnu.no.

${ }^{1}$ See the June-July 2019 Notices, https://www.ams.org/journals /notices/201906/rnoti-p939.pdf

2 https://www.ems-ph.org/journals/news letter/pdf/2019-09-113 . pdf\#page $=23$

For permission to reprint this article, please contact: reprint-permission aams.org.

DOI: https://dx.doi.org/10.1090/noti2049
Dundas and Skau: Professor Uhlenbeck, firstly we want to congratulate you on being awarded the Abel Prize 2019 for your pioneering achievements in geometric partial differential equations, gauge theory and integrable systems, and for the fundamental impact of your work in analysis, geometry and mathematical physics. You will receive the prize tomorrow from His Majesty the King of Norway.

Uhlenbeck: I'm greatly honoured, thank you!

Dundas and Skau: You spent your childhood in New Jersey, and you described yourself both as a tomboy and as a reader. That sounds contradictory, but perhaps it isn't?

Uhlenbeck: I don't believe it is exactly. I think now you would just say that I was interested in sports and the outdoors-"tomboy" is an old-fashioned word-and also, everyone in my family read, so our favourite time during the week was our trip to the library.

Dundas and Skau: Your mother was an artist, and your father was an engineer?

Uhlenbeck: Yes.

Dundas and Skau: Were there strong expectations as to what you and your siblings were to do later in your lives?

Uhlenbeck: Yes, there were strong expectations that we should be able to support ourselves. My parents married in the middle of the Great Depression, and the difficulties with having enough money to live were very present to 
them. So they were mostly concerned that we would actually have jobs. And I think they had expectations of my brother actually getting an engineering degree, engineering being a good profession. As with me they didn't care so much what I did.

Dundas and Skau: You say that you were interested in everything, but you also mentioned that Latin was the only hard course in high school. How did you then end up with mathematics? We would have thought you would have chosen Latin then?

Uhlenbeck: Well, I don't really know myself! It was only lately I got the explanation for myself. But Latin was the only hard subject I had. It was not something you could do right away, you really had to work at translating Latin. You know, to be in this tradition of years and years and years of knowledge, and actually be reading something that was written so long ago was exciting even when I was a youngster. In my last year in high school I signed up for the honours maths course which was calculus. It conflicted with the Latin course, so I signed up for something like Spanish instead. However, after one or two classes in Spanish I changed my mind and I went back to Latin and took the regular maths course, which did not conflict with the Latin course.

Dundas and Skau: Then you enrolled at the university as a physics major?

Uhlenbeck: That's right. I had been turned on to physics. My father was a very intellectual person even though it had nothing to do with his job, and he got books out of the library, and I remember particular books written by Fred Hoyle. I read all those books and I think he also read them. I have to confess that I didn't do all the mathematics in them, but I saw all the mathematics in them. I also remember books by George Gamow that I found in the library. There weren't many books on maths and science in the library at all, so my resources were somewhat limited, but I was fascinated by the physics. Of course, I didn't even know that you could be a mathematician, so I enrolled as a physics major.

Dundas and Skau: So you had some experience with mathematics when you started at the university?

Uhlenbeck: Right. I tell the story all the time, this was three years after the Sputnik ${ }^{3}$ went up, and so there were programmes all over the country in integrated maths and science, encouraging students to study maths and science. So there were honours courses in maths. I took a unified

\footnotetext{
${ }^{3}$ The first artificial satellite, launched on the 4th of October 1957 by the Soviet Union.
}

course in which I had an honours course in maths and an honours course in physics and chemistry, and I just really took to the mathematics right from the very beginning. I enjoyed it and I was caught up in it, and I was actually very good at it. And, you know, when you're very good at a subject, you're also encouraged to go on studying it. I really enjoyed playing with the ideas.

Dundas and Skau: Did you have an aha moment, where you sort of got enthralled by mathematics? You mentioned something about the derivative.

Uhlenbeck: That's right! The first time I really saw a derivative, it was actually not with a professor, but with a teaching assistant for the course, who was doing problem sessions. We hadn't got to taking derivatives in the class, but he showed us how to take a derivative, and he showed us how to take a difference quotient and take a limit. And I still remember that I turned to my fellow student and said: "Are you allowed to do that?" I was very excited to be able to do that. Also, I still remember when I was understanding the proof of the Heine-Borel theorem. I just remember, you know, arguing by using little boxes and things like that. And I was very excited by the experience.

\section{Dundas and Skau: This was at the University of Michigan?}

Uhlenbeck: Yes, I was a first year student there.

Dundas and Skau: The experience at Michigan you describe as sort of special. I suppose you could have gone to other places, but you went to Michigan, and did that turn out to be a good choice?

Uhlenbeck: Yes, it turned out to be a very good choice. Well, they had this honours programme. I also met the right people and the right things happened to me. In my first year at the University of Michigan I earned pocket money by waitressing in the dining hall. I lived in New Jersey so I didn't go home for the break, and I was around. During one of the breaks I was in an art museum. Well, my mother was an artist and I had essentially been going to art museums since I was in the womb; anyway, I was in the art museum, and I bumped into a professor next to me, and it turned out that he was a maths professor. His name was Dan Hughes. He found out who I was and what I did and the first thing I knew-I think it was the next semester, it might have been my second semester, I can't remember when it was-but the first thing I knew was that I was grading linear algebra without having ever taken it! So I was just taken in and, you know, I didn't think about it as anything special. To me I was just somebody who didn't know what was going on and wanted to learn things. But I think I got very good treatment by my maths professors. 
Dundas and Skau: So you were actually seen and you were recognized?

Uhlenbeck: Yes. In fact, I think I took my first graduate course when I was a sophomore. I took the graduate course in algebra and I remember we did the Wedderburn lemmas. I remember that I didn't understand the course, but three years later when I did come to study for the preliminary exams I looked at the material, and I could actually pull it up and understand it. It's amazing what your brain actually does-learning is not linear at all. Anyway, I was already in advanced maths classes as a sophomore. Then I spent my junior year abroad, in München, and I had beautiful lectures. I took lectures from a Professor Rieger and a Professor Stein.

Dundas and Skau: Socially, was that a very different experience than what you were used to from an American university?

Uhlenbeck: The programme that I was in was from the Wayne State University, and there were students from all over the US in that programme. And I remember realising at the time how really good my education at Michigan was. I can tell you, there were students from Princeton, Yale, Columbia and so forth, and I was as well educated, or better educated. Certainly, my mathematical background was much better than the few others in the programme with maths majors. It was also interesting to have rubbed shoulders with American students from all different universities. To your question, the life of a German student was nothing like the life of an American student. You know, I went to the opera, I became enamoured with the theatre when I was there, I learned to ski, and of course I had a German boyfriend at some point. We went for long walks in the Englischer Garten, because it was romantic, and I learned German. Well, I can't say that I know it still but I was pretty good at German at the time that I was there, even though I don't have an ear for language at all.

Dundas and Skau: After the University of Michigan you decided to go on with a PhD-program in mathematics. You spent one year at the Courant Institute in New York and then you moved to Brandeis in Boston because your husband at that time was accepted to Harvard University.

Uhlenbeck: He was a graduate student in biophysics accepted at Harvard, that's right.

Dundas and Skau: But you decided on Brandeis University, even though you may have got into both Harvard and MIT?

Uhlenbeck: I didn't apply. I was already aware of the fact that there were tensions around being a woman in mathematics. And I really wasn't interested in them. NYU (New York
University) had a very special record for women. Lipman Bers had been there and had trained a whole generation of women students. So NYU had a very good reputation towards women. Brandeis hadn't much of a reputation at all. I had an NSF-postdoc and I probably would have got into MIT and Harvard, but some inner radar said not to do that.

Dundas and Skau: You chose Richard Palais as your thesis advisor. Can you tell us why you chose him, and what the theme of your thesis was?

Uhlenbeck: I took a course from him in my first year there. I was a second year student and I was already being noticed, since I came in and passed my preliminary exams. I think maybe only one of the students that had been there for a year did so at the time, the rest all took longer. So whatever feelings there were about having women students disappeared very rapidly at Brandeis. Richard Palais had given this beautiful course on infinite-dimensional topology the year before, but that year he taught a course on the calculus of variations, which is the basis for his book on the calculus of variations and global analysis. I was just excited by this new field. I understood immediately what global analysis was like, and Palais was a beautiful lecturer. I still remember the day I went in and asked him about the heat equation, and he told me everything I needed to know for four to five years. I remember just making this conscious decision I wanted to work in this new field instead of doing a special case of some boundary value problem somewhere. I made a conscious decision to jump in, so to speak.

Dundas and Skau: So the theme was calculus of variations?

Uhlenbeck: That's right.

Dundas and Skau: So this was related to what is called global analysis?

Uhlenbeck: That's right. It was really calculus of variations from the global analysis point of view.

Dundas and Skau: What is global analysis, can you describe it to us?

Uhlenbeck: I think that global analysis was the change from viewing an ordinary or partial differential equation as a very complicated object with lots of indices and with lots of formulas as simple equations in an infinite-dimensional space or an infinite-dimensional manifold. Conceptually, it simplifies what you're doing tremendously. It was also discovered that a whole lot of stuff that you could do in finite dimensions you could do-under the right hypotheses-in infinite dimensions. The typical example would be what the Abel Prize winners Atiyah and Singer did; the 
two actually proved a theorem at that time about partial differential equations. Loosely speaking, it says that a partial differential equation of a certain type has a kernel and a cokernel, like in finite dimensions, and the difference between the kernel and the cokernel comes from topology. This was a very exciting discovery, and it's foundational to the change in perspective towards these equations.

Dundas and Skau: Here is a quote from your article in the Proceedings of the International Congress of Mathematicians in 1990 in Kyoto, where you gave a plenary talk: "In the 1960s, an ambitious subject called 'Global Analysis' developed with the explicit goal of solving non-linear problems via methods from infinite-dimensional differential topology... The optimism of the era of global analysis has ultimately been justified, but this did not happen immediately. The problem is essentially as follows: in order to discover properties of solutions of ordinary or partially differential equations which have global significance, it is essential to make estimates." Could you expand on that?

Uhlenbeck: Let me use something that I have thought of since I started doing interviews. It's a little bit like the question of the large and the small. When you paint a picture you have to have an overall perspective and an overall design and an overall point of view, but the whole thing will fall apart if you can't do the details. Saying "that's a person" is not the same thing as actually making a person out of it carefully with all the skill and background and all the teaching that you have. So the inequalities are the fundamental thing that the global picture is made out of, but in order to know exactly the right ones to do you need the global picture.

Dundas and Skau: An example of that would be to find minimal surfaces in higher dimensions?

Uhlenbeck: That's right. Well, in that case the problem of minimal surfaces turns out to be what you call a borderline case. In my thesis, I actually wrote down quite a few problems in the calculus of variations that satisfied a topological condition in Morse theory called the Palais-Smale Condition. The techniques of manifold theory go through for analysing the gradient flows, and so forth. But the problem is, those are made-up problems. So what happens when you come down to a problem that you really want to solve? In the case of geodesics, the Palais-Smale condition and all the infinite-dimensional stuff go over beautifully just like that, like clockwork. But, of course, we knew how to do geodesics: we just approximated it by broken curves, and reduced it to a finite-dimensional problem. So, the question is, what good is it if it doesn't solve the problem we want to solve? My observation was that if you took the equation that you need to minimize to get a minimal sphere, and you add a small term to it, then it suddenly satisfies the Palais-Smale condition, and Morse theory is true. Now you look at the solutions of that equation and you let the perturbation go to zero, and then you can see what is happening to those solutions. What happens is that those solutions approach a solution, which could be trivial. But there's a place in the surface that you're studying where, as the perturbation goes to zero, all the information collects over that point. So if you take a microscope and look around that point, and you make the area around that point bigger and bigger and bigger, in the limit, you can actually get a solution on the whole plane. And you, lo and behold, notice this in fact solves the whole problem for you because the point at infinity can be added; that's a technical theorem. The point at infinity can be added and you suddenly have found your minimal sphere. You certainly discover that not all the solutions persist, but enough of them persist so you can say something about the problem.

Dundas and Skau: John Nash, who shared the Abel Prize with Louis Nirenberg in 2015, intimated to us in the interview that we had with him that his paper titled "Continuity of solutions of parabolic and elliptic equations" from 1957-58 might have been decisive in him getting the Fields Medal in 1958, except for the fact that De Giorgi, an Italian mathematician, had independently proved that same result at about the same time.

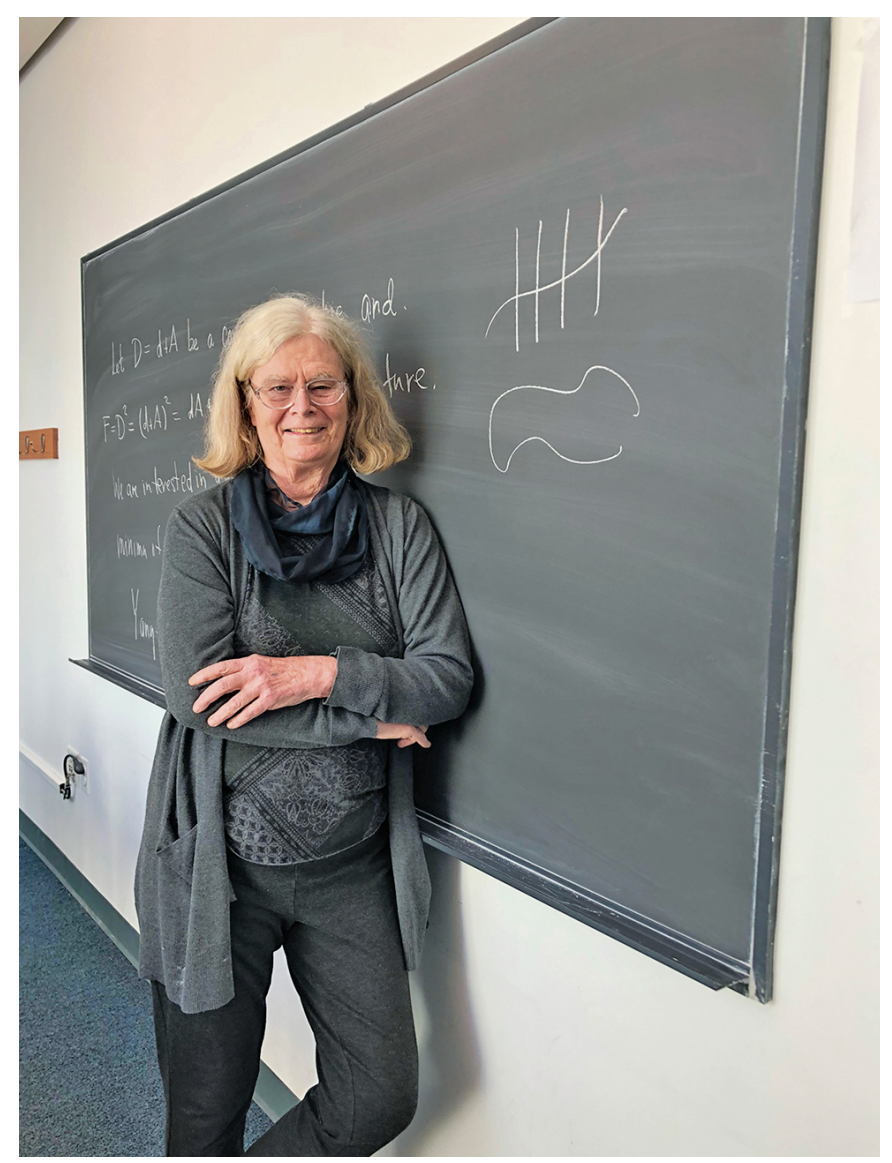

Figure 2. 
In 1977 you published a paper in Acta Mathematica-a prestigious math journal-with the title "Regularity for a class of non-linear elliptic systems." In the introduction you say that the results in that paper are an extension of the De Giorgi-NashMoser result. Could you tell us about this paper and its genesis? Also, the mathematical community took notice of you when your paper appeared. Do you consider this paper to be your first great paper?

Uhlenbeck: Yes, and I still consider it to be my best paper. In fact, it's a very difficult paper and I wonder how easy it would be for me to understand it now. It's a long time ago, it's more than 40 years ago. But the fact is that I had found some calculus of variations problems in my thesis that satisfied Morse theory, so they had a lot of critical points. But the problem is that the geometrically simple cases of them led to integrals that were of a not completely standard sort. It turns out that you could find minima, but these minima were not necessarily smooth; these minima could have singularities. I faced the fact that I needed to show that these minima were actually regular, real solutions, not just what they called weak solutions. I learned enough about the background of the theory when I was a graduate student to be able to show that the derivatives were bounded, but on the other hand I couldn't carry it any further. I worked and fussed with this problem for a long time. In fact, if it had just been one function that you minimized, the De Giorgi-Nash-Moser result would have given that the solutions were regular. By the way, Moser's name has been tacked on to the De Giorgi-Nash theorem since he simplified their proofs. But I had a system, that is many functions, and those techniques didn't a priori carry over. I had actually met Jürgen Moser and he sent me some of his reprints, which I read very carefully. At some point I was able to use his Harnack inequalities to actually prove the fact that those solutions are regular. Actually, they have critical points where the derivative of the function vanishes, but I was able to see that the functions were smooth enough, as smooth as you would expect of them. I remember S.-T. Yau having me come to California to see him and talk to him about that paper. There I also met Leon Simon and Richard Schoen.

Dundas and Skau: This particular paper was on partial differential equations and had very little to do with geometry, right?

Uhlenbeck: Actually, it had nothing to do with geometry.

Dundas and Skau: The techniques you developed in this paper, were they important for you when you wrote papers later?

Uhlenbeck: Actually, no! Well, I'm afraid that's a little bit of the story of my mathematical career. I could have pursued it and made extensions of it and carried it further. There were some non-trivial points about putting more variables in there, and I kind of figured out how to do it. I saw you could do it but I didn't know if it was going to be useful, and I still don't know whether that problem is useful. So I didn't pursue it, but someone else did-Martin Fuchs did, actually.

Dundas and Skau: But then we are entering a different phase in the beginning of the 1980s, where you published a series of highly influential papers. Those must have been amazing years? What were the conditions that made this possible?

Uhlenbeck: I don't really know, because I was getting a divorce. I moved from the University of Illinois at UrbanaChampaign to the University of Illinois in Chicago. I got together with a new boyfriend, and I taught at the University of Illinois in Chicago. I taught two courses a term for three terms, three quarters a year, and I think I travelled a lot. So, how I did this I have no idea! I find it amazing!

Dundas and Skau: They sound like horrible conditions!

Uhlenbeck: I like to tell this, because young people really think that you have to be at Oxford or some other prestigious place to actually do good work, and I think there's no evidence that that's really true.

Dundas and Skau: One of the first papers in this remarkable series is co-authored with Jonathan Sacks and is titled: "The existence of minimal immersions of the 2-spheres." There you develop a series of techniques, both with respect to regularity and with respect to compactness. We couldn't find the term in the paper, but is it here the "bubbling" idea starts?

Uhlenbeck: Well, I asked Jonathan Sacks about where that idea comes from, and he thinks that I actually used it in some talks I gave about the theorem. The technique is in the paper but we didn't call it anything, and I think I only used it in talks. But the name caught on.

Dundas and Skau: And there you're studying immersed 2-spheres modulo the action of the fundamental group, and you're saying that you can represent them by particularly nice immersed spheres. There were certain technical things there that you encountered that gave rise to these bubbling effects, right?

Uhlenbeck: Yes, right. The idea is that you add on a small term-you can do this to most problems actually-and then it satisfies the Palais-Smale condition. It allows you to construct a Morse theory. And that gives you lots of solutions, lots of minima, lots of saddle points. But now you really want solutions to the original problem, not to the approximate problem, so you want to take the perturbation away. Now this works best in the scale invariant case, 
meaning that the problem does not really see scales. So what happens is that you take the limit and get a solution. But the solution might actually be trivial, it might just be a map to a point. But you go back and see what happens to the solution and it actually converges everywhere, except at a finite number of points. And around these points what happens is a scaling invariant problem. So the little region around the point thinks it's just as good as the big plane. And so you have the description of a solution as a "bubble," a sphere, actually happening around a little tiny point. And by looking at it with a microscope, a magnifying glass, and blowing it up-I think I used the term "blowing it up" at this time-you see the bubble that happens at that point and you get a solution on a plane. Then you prove that can put the point at infinity in. That's a regularity thing, namely that you can put the point at infinity in. So that way you could actually construct quite a few of these immersed spheres.

Dundas and Skau: Indeed, it's a generating set for $\pi_{2^{\prime}}$ is it not?

Uhlenbeck: I think someone else proved that. Actually, this is one of the things we saw we could do but we didn't do it.

Dundas and Skau: These bubbles that occur, how do you control that there aren't infinitely many of them?

Uhlenbeck: That's an estimate. Well, actually, the answer is: each bubble needs a certain amount of energy, and you have only a finite energy. If you want you could even make an estimate of how many you can have at most.

Dundas and Skau: It is hard to choose, but many people hold your two papers from 1982 titled, respectively, "Removable singularities in Yang-Mills fields" and "Connections with L bounds on curvature" in particularly high esteem. Could you give us a brief overview? Specifically, why are the Yang-Mills equations important, and why is gauge invariance important?

Uhlenbeck: Well, the Yang-Mills equations are important because high energy theoretical physicists told us they were important! Mathematicians could very well have done the whole theory, they just didn't think of doing it. So, it's one of these pieces of evidence that pure mathematics really needs input from outside of itself. Sometimes it's even another branch of mathematics that can give valuable input, but this is an example of ideas outside mathematics that turn out to be important in mathematics. Physicists actually got very excited about mathematics, probably because this was an application of the Atiyah-Singer index theorem to tell you what the dimension of the space of the solutions was.

It's a topological invariant, and it needed ideas from the nascent field of global analysis. They, i.e. the physicists, had explicit solutions of a certain type on the four-dimensional sphere, solutions that they could explicitly write down. And they had some more complicated ones that they could write down. However, they knew they didn't know all the solutions of the more complicated sort by the Atiyah-Singer index theorem, and so it becomes a question about what the spaces of solutions of such things look like. And the removable singularity theorem from the first paper comes from the fact that if you take a sequence of these solutions, and if it doesn't converge to a solution, you know that it converges to a solution off a particular point. And at that point the bubbling phenomena happens. So, my first theorem about removable singularity was proving that you can put in that point where the solutions fail to converge.

The second paper is a little bit different. The Yang-Mills equations themselves are not elliptic equations, basically due to the presence of a large symmetry group. They have a coordinate invariance. It's like you're looking at a plane and you're not using Cartesian coordinates, but you're using any arbitrary set of coordinates you want to write down. You still have a plane, but you have all these ways to describe it. So what happens in gauge theory is that you have these physical objects called connections. I think they call them fields in physics; mathematicians call them connections. They have this gauge invariance, which means that they have coordinates that are free. And there are way too many of them - they correspond to a symmetry group-and you have to divide out by them. The problem is that you have to do something rigid, like constructing Euclidean coordinates on them. And what I did, I showed under what circumstances you can actually construct these coordinates. Once you have the right coordinate system you just treat it from standard PDE methods. That's described in the book Instantons and Four-Manifolds that I wrote with Daniel Freed, but you need the second variate equation. Someone had to do it. I have to say this is one example of if I hadn't done it, someone else would have done it. I mean it had to be done.

Dundas and Skau: We are now talking about the Yang-Mills equations and gauge theory, which first popped up in physics, but had tremendous influence on mathematics. Many of us are familiar with the article from 1960 by the physicist and the 1963 Nobel Prize recipient, Eugene Wigner, with the intriguing title "The unreasonable effectiveness of mathematics in the natural sciences." Considering what has happened in global analysis we could perhaps turn this on its head and say: "The unreasonable effect that physics has had on mathematics"?

Uhlenbeck: No! Well, I don't know about the Greeks, but certainly there was actually no difference between maths and physics with for example Isaac Newton. In fact, the real division between maths and physics occurred in the 19th century, where people like Weierstrass started putting all sorts of holes in the arguments that people were making. 
They were saying: "Okay, you take a sequence of things, how do you know that there is a minimum, maybe there isn't any minimum, you physicists are assuming there is." So you get a real division. Mathematics kind of separated itself because it needed the foundation of rigour. I mean, you can see this happening with infinite-dimensional vector spaces becoming very important. In the theory of calculus of variations the most important space is called a Hilbert space, so that would date that for you. And they are absolutely essential in quantum mechanics. Physicists were the ones who introduced Dirac's deltas and so forth. But mathematics had to separate and make all this rigorous before you could actually have a mathematical subject. So you see a real division occurring between maths and physics at this point. Maths kind of separated itself and made things robust and rigorous. The physicists weren't really interested in this, and actually the mathematicians stopped being interested in physics, too. And then I think it came back together at some point.

Dundas and Skau: The example you mention is very interesting. Weierstrass pointed out that Riemann did not have a rigorous proof that the so-called Dirichlet problem had a solution. In fact, Riemann's defective proof relied on a kind of minimizing procedure that he called the Dirichlet principle.

Uhlenbeck: That's right!

Dundas and Skau: Also, Riemann was certainly a more physics-inclined mathematician than Weierstrass was.

Uhlenbeck: I see. I didn't actually realise that. I don't really know so much about that part of the history.

Dundas and Skau: But then, of course, your results from these two papers are taken further. At this time you're an established mathematician, and you're seeing that people like Taubes, Freedman, and Donaldson are grabbing hold of the things you are doing and proving remarkable things about four manifolds. We really don't see the connections with what the physicists were originally thinking about. Could you elaborate on that?

Uhlenbeck: Well, Taubes' PhD thesis is in physics, and as a graduate student he wrote a book called Vortices and Monopoles with his advisor Arthur Jaffe. Some of that is motivated by the connections with physics and, in fact, one of the hot topics in that subject right now is Higgs bundles. A physicist at my department at the University of Texas, Andrew Neitzke, is studying them just as hard as any mathematician would have done, so I don't know how much they are separated. But certainly they started to have a life of their own in mathematics.
Dundas and Skau: A quite spectacular life at that. Could you give a short outline of the dramatic developments in four manifold theory that ensued and in what parts your contributions were particularly important?

Uhlenbeck: Donaldson's classification of simply connected four manifolds with definite intersection form is based on the construction of the boundary of the moduli space of solutions to the self dual Yang-Mills equations. There are a number of ingredients in this construction. First of all, my theory on bubbling occurring in limits of solutions to the Yang-Mills equations shows that the boundary consists of lower-dimensional solution spaces with bubbles attached. Cliff Taubes shows which of these configurations occur as limits of smooth solutions. In the simplest case, the boundary consists of the four manifold itself, and the moduli space provides a cobordism of the manifold with a neighbourhood of the singular points of the moduli space. Hence, not all the continuous four manifolds constructed by Freedman can be given smooth structures. In fact, none of the exotic examples can be smoothed.

Dundas and Skau: With R. Schoen you prove that any minimizing map from a Riemannian manifold to a compact Riemannian manifold is smooth outside a closed bounded set of codimension three. Could you tell us about this result and why the singular set grows with the dimension?

Uhlenbeck: The work of Schoen and myself on harmonics maps, and in fact all the theorems of this type, is based on monotonicity, which estimates energy in small balls in terms of energy in larger balls. We show that when the scaled energy is sufficiently small, the solution is smooth. So singularities need a certain amount of energy. A counting argument shows that the singularities can only form on a set of Hausdorff codimension two (it is four for Yang-Mills). It can be tricky to actually get this down to codimension three, but the argument depends on both monotonicity and an approximation to the blow-up of the singularity, which is a harmonic map from $S^{n-1}$ into the target manifold.

Dundas and Skau: The conventional picture is often that a good mathematician is a person with really outstanding intellectual power, who solves the problem through his or her superior genius, and the solution comes as a kind of bolt of lightning. We know of course that this is not the typical case...

Uhlenbeck: Well, there's a lot of luck involved. There's a lot of knowledge of how to take advantage of luck!

Dundas and Skau: Right! However, for most of us the most important quality-besides of course a good intellectual capacity-is perseverance and the capacity of concentration. Could you expand on this and, also, have you had moments of epiphanies, 
where in a flash you saw solutions to problems you had been struggling with?

Uhlenbeck: Let me answer your last question first, and the answer is yes. You struggle with a problem, it can be over a period of years, and you suddenly get some insight. You're suddenly seeing it from a different point of view and you say: "My goodness, it has to be like that." You may think all along that it has to be like that, but you don't see why, and then suddenly at some moment you see why it is true. It could also be a very simple idea that suddenly hits you. I don't remember where I was and what I was doing when I had those moments, but I still remember those moments.

Dundas and Skau: But in all these cases we are talking about a moment, like a bolt of lightning?

Uhlenbeck: There is a moment when you suddenly realise that you see how to do it. And that of course comes after all the struggle you had. Struggle isn't the right word, because you wouldn't do it if it wasn't also a lot of fun, all the time you spent thinking about this problem. Then, of course, you get the problem that is even worse: you have to write it up! But there is this moment right in between when it's really great. I remember these moments, but I have to tell you, suddenly when everything fits together you keep going back and checking if it's right. In fact, I had a similar moment a year or so ago about a problem I'd started working on, and I kept on going back and checking it to be sure that it was right. As to the first part of your question, I think you can't do mathematics without the ability to concentrate. But also, that's where the fun is, the rest of the world fades away and it's you and the mathematics. And I think there isn't any other way to do mathematics.

Dundas and Skau: And, of course, that's one of the reasons that there are so few mathematicians. It's a very special endeavour appealing to a small minority whose minds are wired in a special way.

Uhlenbeck: Well, I also think society doesn't have the will to support too many mathematicians. You mentioned perseverance, but you know, it's also an escape! Some of us really see it as an escape.

Dundas and Skau: But there are two sides to this. The community is important. We promised to come back to Yau, who was one of the mathematicians that really believed in you. That kind of recognition can be crucial as well.

Uhlenbeck: Yes, the community is very important. Yau is a brilliant mathematician, but he is also good at inspiring students and other people. He is good at finding mathematical results that he likes and getting other people involved in them.

Dundas and Skau: In 1985 you published a paper together with Yau titled "On the existence of Hermitian-Yang-Mills connections in stable vector bundles." This work had a profound impact on the field of complex geometry as well as in physics. Edward Witten declared that the Hermitian-Yang-Mills is one of the major building blocks of supersymmetric string theory, and it provides a very elegant existence theorem by reducing to a criterion in terms of purely algebraic geometry. How did this work with Yau come about?

Uhlenbeck: I knew from the late 1970s that Yau admired my mathematics. Also, Richard Schoen and Yau, and Jonathan Sacks and I, published essentially the same paper about minimal immersions of Riemann surfaces. These are minimal objects of different shapes like a 2-sphere, a torus, a two-holed torus or something. Anyway, Yau approached me and told me the problem. I didn't know anything about the field at all. One of the problems was to find out what the stability condition means. There is also a different formulation in terms of complex geometry than in real geometry. I was able to absorb this and my contribution was like what I'd done before: I added an epsilon and a term that made the problem solvable. You solved that problem, and then you took the perturbation away and looked at limits. And again you are faced with the same problem as before: you have to know what the limits look like. And that was the hard part of the paper, actually.

Dundas and Skau: After 1989 you produced a series of papers about harmonic maps into symmetric spaces where the action of the loop group features prominently. Could you tell us about this project and also about your later collaboration with L. Terng and L.M. and R. J. Siebner?

Uhlenbeck: I can't really go into the work with Terng in less than ten pages. It is somewhat accidental that this example

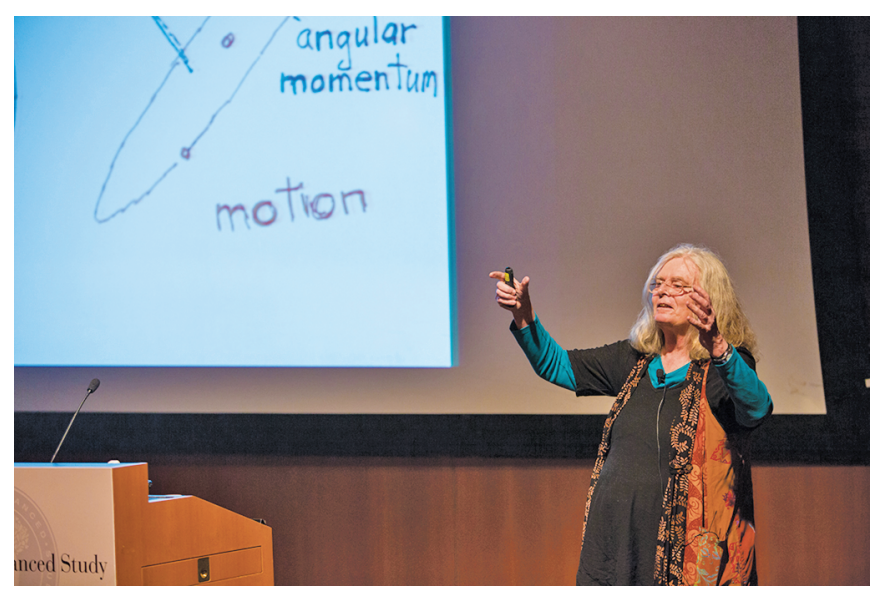

Figure 3. 
has to do with harmonic maps, as our work is on a number of different equations: KdV, non-linear Schrödinger and Sine-Gordon equations. I also don't really want to describe the work with Siebner and Siebner. It is based on a mountain pass lemma, and a loop in the space of connections which is not contractible, but which does not have enough energy to allow bubbling under gradient descent.

Dundas and Skau: You coined the expression "linear thinking versus sloppy thinking" to describe two types of mathematicians. One type thinks linearly, step by step, while the other is a more intuitive type of mathematician. One type tends to be a theory builder, while the other tends to be a problem solver. You count yourself as a problem solver, right?

Uhlenbeck: Yes, I definitely belong to the problem solvers. I'm really not much of a theory builder at all. In fact, I don't even read papers that way, I don't read papers from start to finish. I look at the beginning, I look at the end, I look at the references, I try to find the main theorems, I try to find the definitions, and then I try to find the key lemmas. Then I try to prove the key lemmas, and when I get stuck on the key lemmas I go back and look at the paper. That's a typical scenario for the way I read a paper. So that might give you some idea why I do not build theories!

Dundas and Skau: You have said, and we quote: "I have an addiction to intellectual excitement, and as a consequence I find that I am bored with anything I understand." Could you expand on that? Specifically, does this have as an effect that you have shied away from conventional problems, so to speak, and rather focused on problems arising in new and unchartered territory?

Uhlenbeck: I had the privilege of working in several fields (eigenvalue problems, harmonics maps, gauge theory, integrable systems) when very basic ideas were being developed. I know from going to seminars that these subjects have developed a great deal, with many more examples and details worked out. I find I am not interested or excited by the new results as I was when the subjects were new and basic ideas were being worked out. I regard this as an intellectual failing, and as far as I can identify my thoughts on my career, it is the one single regret I have. Some of my students have suffered, as I gave new problems in new fields to many of them, but then did not help advertise their work among my colleagues or help them develop their ideas as I might have. In an alternative life I might have contributed more to the development of mathematics instead of always looking for new directions and different approaches.

Dundas and Skau: Hilbert in his talk at the ICM congress in 1900 in Paris, where he presented his famous 23 problems, said the following: "As long as a branch of science offers an abundance of problems, so long is it alive; a lack of problems foreshadows

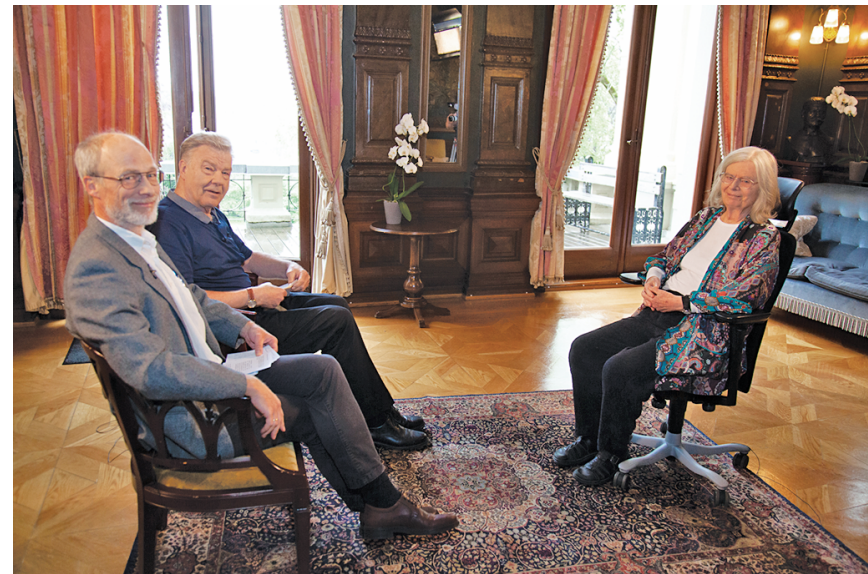

Figure 4. From left to right: Bjørn Ian Dundas, Christian Skau, and Karen Uhlenbeck.

extinction." How does global analysis, in particular gauge theory, fare with respect to Hilbert's statement? Are there still big problems around?

Uhlenbeck: Well, a lot of the areas in which you really would like to understand the problems are very difficult to access and one is quite stuck. For example, there are lots of problems which have to do with complex gauge groups. The gauge theory that I was talking about all had to do with things like unitary groups and special unitary groups. You can actually look at special linear groups, so there is an imaginary part of the connection which accesses the Higgs field. Clifford Taubes spent a lot of time trying to understand those problems. The two-dimensional case of Higgs bundles has actually been a very hot area of research over the last five or six years. It's a big open problem how to think of these things, because the limits of solutions have singularities, and it is always very difficult to understand singularities. So that's a big open problem. My answer to you about what the big open problems are is that we would all be there if we knew what they were! I haven't been that active mathematically the past decade, so I don't know if I'm the right person to ask. These days I count on other people bringing me good problems.

Dundas and Skau: But in 1988 you did make some predictions.

\section{Uhlenbeck: Oh dear!}

Dundas and Skau: In "Instantons and their relatives" you list five key points that you were thinking mathematics was moving towards. One was "Simplicity through complexity." We think that was about moduli?

Uhlenbeck: Yes, that's right!

Dundas and Skau: Do you feel that mathematics has gone in that direction? 
Uhlenbeck: Yeah, I think so. There are a lot of topological invariants that are constructed using models that were originally a gauge group and an associated Higgs bundle. They are very complicated because there are a lot of fields and a lot of different terms. But when you actually write down the equations you are trying to solve and look at the space of solutions, the moduli space is actually very simple. So I think it's fair to answer yes to your question.

Dundas and Skau: And you asked, just as a question: "And after theoretical physics?", indicating that you think that in the future inspiration will come not only from physics, but also from other sciences.

Uhlenbeck: Well, I'm thinking it ought to be, because certainly the field of mathematical biology has grown, and the field of computer science has a lot of interesting aspects that must have mathematical connotation, and so forth.

Dundas and Skau: You say another interesting thing: "I hope that no comments on the place of women in mathematics are even relevant by 2038."

Uhlenbeck: Yes, right, I really hope that.

Dundas and Skau: Continuing in that vein for a short moment: reflecting on your own experience, you say in an interview with the New Yorker: "I figure, if I had been five years older, I could not have become a mathematician because disapproval would be so strong." Could you expand on that?

Uhlenbeck: I became a mathematician in the wake of the second wave of feminism; actually as a result of Sputnik and the second wave of feminism. The point is that Betty Friedan's book The Feminine Mystique opened up people's eyes to the fact that a lot of life was not open to women. So this is in the early 1960s and I was already at school at this time. By the time that I got into graduate school, and then looked for a job, the fact that women might do something else was actually in discussion. Five years before it probably wasn't really in discussion that women might be doing something like this. And I feel that the combination of Sputnik and the second feminist movement really paved the way and opened the doors for me. Five years earlier I would have missed that.

Dundas and Skau: You have said that you were respected by your immediate mathematical colleagues, who recognized the brilliant mathematics you were doing. But the broader community was less accepting, some being very sceptical. Do we see a parallel here to how your most famous female predecessor, Emmy Noether (1882-1935), was treated way earlier?
Uhlenbeck: Well, something changed, but it changed in the 1960s. Success for women in traditionally male fields is a complex issue.

Dundas and Skau: Emmy Noether was one of the great mathematicians of the 20th century, and she is arguably the greatest female mathematician of all time. She gave a plenary talk at the ICM (International Congress of Mathematicians) meeting in Zürich in 1932. The next time a woman was invited to give a plenary talk at an ICM meeting was in Kyoto in 1990, and that woman was you. You are also the first woman to be a recipient of the Abel Prize-altogether there are now 20 recipients. In 2014 Maryam Mirzakhani became the first woman to get the Fields Medal-60 Fields Medals have been awarded so far. What this dramatically illustrates is that mathematics has been dominated by men. The topic women and mathematics is a many-faced issue involving cultural factors, stereotypes, prejudice and much more, and we will not get into that. However, we would like you to respond and confront directly those beliefs and viewpoints that still linger, and which can be summarized as follows: the reason mathematics is so dominated by men is for a large part due to the fact that men are predisposed to abstract thinking, and, in particular, to mathematics.

Uhlenbeck: Absolutely not! I don't believe that at all. Among other things, it's not even clear what you need to be a good mathematician. The more diverse population you have doing something like mathematics the better it is. So I think that is a completely misguided viewpoint. The fact that there were no women mathematicians was because they couldn't study, they couldn't get jobs, and they had a hard time getting respect from all but their immediate colleagues. On top of that, the other women didn't accept them, we even had to struggle with that. That changed over a period of years and there's no question in my mind that things have really improved, at least in the United States. My understanding from talking to people is that it has not improved everywhere. And it's still true that women in their 50s have a tough time. But the younger women seem to have found acceptance and openness. The community has changed. The younger women are more visible, they are more talkative, and they are more involved in the community. I like to think that things have really changed, but many people don't realise what it was like in the 1960s.

Dundas and Skau: So you think that the glass ceiling has been broken?

Uhlenbeck: Well, yes... yes and no. I wouldn't say that there aren't problems, it's just a lot better than it was.

Dundas and Skau: You voice concern for minorities in mathematics, as well, and you did that again when you were told that you were going to get the Abel Prize. Is that something you have 
been concerned about for a long time? Is that related to other initiatives that you've undertaken?

Uhlenbeck: Actually, quite a few people are concerned about it. The fact is that-perhaps not when I was younger, but by the 1990s when I started to help women-I became aware of the difficulties that under-represented minority students have. I also knew Elisa Armendariz very well, who was our chairman at the University of Texas for many years, and who is Hispanic. Many people are concerned about the difficulties that minority mathematicians have. The question is what you can do about it. The problem is, one doesn't know what to do about it.

Dundas and Skau: But you had initiatives like the Park City Mathematics Institute.

Uhlenbeck: The story of Park City is tied up with how I got involved with the women's programme. When I founded it, I thought, this is great, we'll have Park City and there will be a handful of women mathematicians showing up, and we will all get together and know each other. The problem was that there weren't even a handful of women that showed up, it was so predominantly male. So that was when I became involved with women. And basically I got involved because the Institute for Advanced Study gave me money, secretarial support, and the prestige to actually try to start a programme. And I had Chun-Lian Terng as collaborator, and we could do maths-or we thought we could do maths at the same time that we did this organisation-and we actually did a little maths throughout this. So, you know, when I see an opportunity, then I'll try to do something.

Dundas and Skau: Could we ask you what you plan to do with the prize money?

Uhlenbeck: When I learned that I had got the prize I was of course amazed, overwhelmed, and so forth. But the very next day, before it was even publicly announced, somebody said: what are you going to do with the money? And I said: money? I hadn't thought about that yet. But I thought about it and I realised that I wanted to do something for under-represented minorities. And I wanted to do something that is going to work! I don't want to just go out there and do anything. So I called up my friend Rhonda Hughes, who I knew from the women's programme. She has been running an EDGE-programme (EDGE stands for Enhancing Diversity in Graduate Education). She and Sylvia Bozeman from Spelman College have been running a programme for graduate students who are just starting out, half of which are minority women, and they share excellent ties with the minority maths community. I called her up and talked to her, and I made the decision that I'll give half of the prize money away. One third of it will go to the Institute for
Advanced Study and two thirds of it will go to the EDGE foundation, which gives scholarships to minority students. The Institute for Advanced Study has already matched the money that I'll be giving them for this purpose, so I'm very pleased about that.

Dundas and Skau: That's splendid. We end this interview by asking what interests and hobbies you have outside mathematics?

Uhlenbeck: Walking in the mountains would be at the top of the list. I've started to paint a little bit. Actually, I was not so well for a while, and I started to play the recorder again, and I started doing some painting. At this age I have to keep up my exercise programme and keep up with my friends. I find that life is already very full.

Dundas and Skau: On behalf of the Norwegian and European Mathematical Societies, and the two of us, we thank you for this very interesting interview. And again, congratulations on being awarded the Abel Prize.

Uhlenbeck: Thank you. I am deeply honoured.

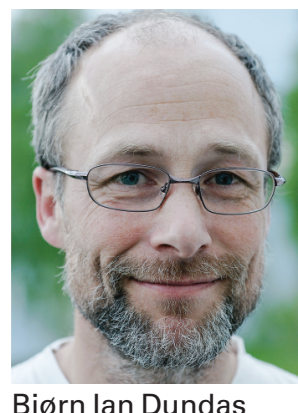

Bjørn lan Dundas

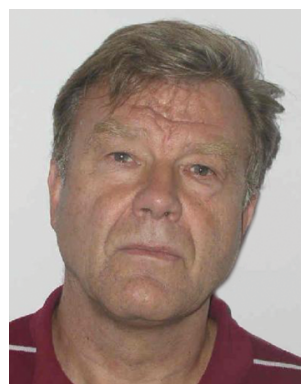

Christian Skau

\section{Credits}

Figures 1 and 3 are ${ }^{\odot}$ Andrea Kane/Institute for Advanced Study.

Figure 2 is ${ }^{\circledR}$ Lee Sandberg/Institute for Advanced Study.

Figure 4 is ${ }^{\odot}$ Eirik Furu Baardsen/DNVA.

Photo of Bjørn Ian Dundas is by Halvard Fausk.

Photo of Christian Skau is courtesy of Christian Skau. 